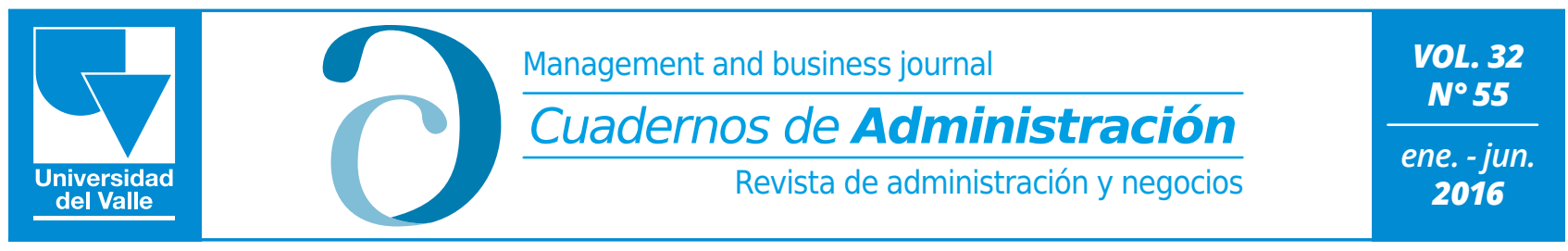

Print ISSN: 0120-4645 / E-ISSN: 2256-5078 / Short name: cuad.adm. / Pages: 7-18

\title{
Propuesta de un modelo de medición de la calidad y su impacto sobre la riqueza
}

Proposal for a model of quality measurement and its impact on wealth Proposition d'un modèle de mesure de la qualité et son impact sur la richesse

\section{Luis Alberto Pérez Bonfantea}

Profesor Tiempo Completo, Facultad de Ciencias de la Administración, Universidad del Valle, Cali, Colombia.

E-mail: luis.alberto.perez@correounivalle.edu.co

\section{Resumen}

Research article, PUBLINDEX-COLCIENCIAS clasification Submmit: 06/02/2016

Review: 23/03/2016

Accepted: 15/06/2016

Eje temático: calidad y marketing

La calidad, de acuerdo con sus implementaciones y publicaciones, se ha convertido en una herramienta para el mejoramiento empresarial. En la misma vía, el valor económico agregado [economic value added, por sus siglas en inglés (EVA)] se ha venido constituyendo en un potente mecanismo para la gestión y exposición de sus resultados económicos. La propuesta que se presenta en este artículo hace una combinación de indicadores para medir la gestión de la Calidad Efectiva Operacional (CEO) y su articulación con EVA, contribuyendo así al desarrollo de la riqueza de los dueños de la empresa, característica del modelo neoliberal. El artículo se ha estructurado a través de tres apartados. En el primero, se exponen los elementos de orden conceptual respecto a la calidad y la gestión del EVA; en el segundo, se describe el modelo para la medición de la Calidad Efectiva Operacional, junto a su implementación; finalmente, se formula una serie de conclusiones que impulsan a seguir investigando las temáticas y su articulación, en aras de aportar hacia la construcción de organizaciones más competitivas.

Palabras clave: Calidad, Calidad económica, Valor agregado.

\begin{abstract}
Quality, according to its implementations and publications, has become a tool for business improvement. In the same way, the economic value added (EVA) is becoming a powerful mechanism for managing and exposure of its economic performance. The proposal presented in this article makes a combination of indicators to measure the management of the Effective Operational Quality (CEO, for its initials in Spanish) and its articulation with EVA, thus contributing to the development of the wealth of the company's owners, characteristic of the neoliberal model. The article is structured through three sections. In the first one, the elements of conceptual order regarding the quality and management of the EVA are exposed; in the second section, it is described the model for measuring Effective Operational Quality, along with its implementation; finally, a series of conclusions are formulated, which drive to keep investigating the issues and their articulation, in order to contribute towards building more competitive organizations.
\end{abstract}

Keywords: quality, economic quality, added value.

\section{Résumé}

La qualité, en fonction de son implémentation et de ses publications, est devenue un outil pour l'amélioration des entreprises. De la même façon, la valeur économique ajoutée [economic value added en anglais (EVA)], se constitue comme un puissant mécanisme pour la gestion et l'exposition des résultats

a Ingeniero Industrial, Magíster en Administración de Empresas, Universidad del Valle, Cali, Colombia. 
économiques. La proposition présentée dans cet article utilise une combinaison d'indicateurs pour mesurer la gestion de la Qualité-Efficacité Opérationnelle et son articulation avec I'EVA, contribuant ainsi au développement et à la croissance de la richesse des propriétaires de l'entreprise, caractéristique du modèle néolibéral. L'article s'est structuré à travers trois sections. Dans la première, s'exposent les éléments d'ordre conceptuel portant sur la qualité et la gestion de l'EVA ; la deuxième, décrit le modèle pour mesurer la Qualité-Efficacité Opérationnelle, ainsi que sa mise en œuvre ; finalement, on formule une série de conclusions qui visent à continuer la recherche des thématiques et leur articulation, afin de contribuer à la construction d'organisations plus compétitives.

Mots clés: Qualité, Qualité économique, Valeur ajoutée.

\section{Introducción}

La calidad, a lo largo de los siglos XX y XXI, ha marcado especial orientación hacia la consecución de resultados que han de impactar la dimensión económica de las empresas, para los que ha desarrollado diferentes enfoques, metodologías e instrumentos de orden administrativo y técnico-operativo, destacándose entre otros, normas técnicas (certificaciones de calidad), modelos de excelencia en gestión (premios a la calidad), herramientas estadísticas. (Aldana, 2010).

Sin embargo, y como lo señalan Guasch et al. (2008) ${ }^{1}$, y el Centro Nacional de Productividad (2005) aún no es claro el resultado de la calidad en la dimensión económica de las empresas. Además y con las nuevas tendencias en la gestión organizacional, dentro de ellas el economic value added (EVA), se hace necesario avanzar en la construcción de mecanismos que permitan medir la calidad y su efecto económico.

\section{Marco conceptual}

Después a la II Guerra Mundial, Japón emprende el proceso de recuperación económica en la que participan diferentes personalidades de Estados Unidos como Edward Deming y Joseph Juran, quienes enseñan aspectos técnicos de la calidad para la reducción de la variación en los procesos y el incremento en la conformidad del producto a fin de aumentar la satisfacción del cliente y ampliar la participación en el mercado de manera sostenida. Además, desarrollan, a través de la implementación de la calidad en su dimensión técnica, aspectos de orden administrativo que se convierten en la década de 1980 en la revolución administrativa de las empresas en los Estados Unidos (Deming, 1989).
El proceso emprendido en Japón genera un impulso importante en el desarrollo de la calidad. Hasta ese momento (1940 a 1950), la calidad estaba concebida principalmente como un proceso técnico de inspección basado en los estándares británicos (tablas militar estándar). Así se generan cambios en la parte técnica (nuevas herramientas, metodologías y demás) y se inicia la concepción de la calidad como un asunto también de orden administrativo (Deming, 1989). Se puede observar que en la década de 1980 toma relevancia significativa en los EE.UU., pues, Japón ya estaba dominando algunos sectores de la industria como la automotriz.

Por otro lado, en la transformación industrial de Japón, Ishikawa (1997) señala que “Desde 1960 el Japón entró en la era de liberalización comercial. En 1962 se fijó un plan de largo plazo cuya meta era liberalizar el 88\% del comercio. Algunos ejecutivos desaprobaron el plan, pero quienes nos ocupábamos del control de calidad apoyamos decididamente la liberalización. No había que temer a la liberalización siempre y cuando el Japón siguiera produciendo artículos de alta calidad y bajo costo". Además, Ishikawa apoya su planteamiento en las ideas de Feigenbaum (2004) que define el control total de la calidad (CTC) como "un sistema eficaz para integrar los esfuerzos en materia de desarrollo de calidad, mantenimiento de calidad y mejoramiento de calidad realizados por diferentes grupos en una organización, de modo que sea posible producir bienes y servicios a los niveles más económicos y que sean compatibles con la plena satisfacción de los clientes" (Ishikawa, 1997).

A los aportes de estas personalidades se suman otros como los de Deming (1989). Él desarrolla la reacción en cadena, a través de la cual señala "mejorar la calidad, produce la

1 Impacto de la certificación de sistemas de gestión de la calidad en las empresas colombianas. 
reducción de los costos, estos a su vez mejoran la productividad y ello permite capturar el mercado con mejor calidad y precios más bajos" (Walton, 1997). Escalante (2006), basado en las ideas de Taguchi (1987), señala que: "Un sinónimo de calidad es uniformidad o antivariación, es decir, existe una relación inversa entre calidad y variación: a menor variación, mayor calidad". Por último, Taguchi (1987) define a la calidad como "pérdida que un producto causa a la sociedad", es decir, calidad significa pérdida cero (Escalante, 2006).

Así, entonces, la calidad se expone como un ejercicio sistemático en dos dimensiones conectadas: la satisfacción del cliente a través de productos y servicios conformes y la reducción del costo por variación en los procesos, lo que se expone de mejor manera en la historia automotriz japonesa, en contraste con la de EE.UU.

\subsection{Modelos y herramientas en calidad}

Los efectos económicos de la satisfacción del cliente y la reducción de la variación, factores que como se verá más adelante, están incluidos en el EVA, hacen mención a aspectos administrativos y técnicos de la calidad como:

- Aspectos administrativos de la calidad. En esta dimensión es importante anotar elementos de sesgo cualitativo de la calidad relacionados con la planeación y la cultura empresarial, en los que diferentes personalidades e instituciones a lo largo de la historia del siglo XX han hecho énfasis. Walton (1997) señala el método gerencial de Deming (1989) (los 14 puntos, las siete enfermedades mortales y algunos obstáculos) a través del cual se describe el papel de la gerencia en el logro de la calidad y su mejoramiento continuo. Otro aporte lo brinda Ishikawa (1997) cuando expone en el marco de las características del control de calidad japonés, 14 ideas asociadas con las actividades de calidad realizadas en Japón con respecto a EE.UU. y Europa Occidental, estableciendo el factor sociocultural como fundamento de las mismas. Asimismo, Escalante (2006) citando a Feigenbaum (2004), señala 10 recomendaciones de este para el éxito de la calidad total, entre las que se destacan: la calidad es una forma de administrar, la calidad es el camino con menor inversión de capital y de mejor costo efectivo para elevar la productividad y, la calidad es un proceso global en la compañía. Por último, Crosby, citado también por Escalante (2006), expone el plan de "cero defectos" de dicho autor que está basado en 14 puntos dentro de los que están: evaluación del costo de la falta de calidad, corrección de causas de error y establecimiento de metas de mejoramiento.

Adicionalmente a estas personalidades, existen múltiples instituciones que abordan los aspectos de la satisfacción del cliente y reducción de los costos, mediante modelos de gestión reconocidos como premios, por ejemplo, premios a la calidad. Inicialmente, la Fundación Europea para la Administración de la Calidad (European Foundation for Quality Management, EFQM, por su sigla en inglés) es una institución creada por un conjunto de grandes compañías europeas en 1988 y que desde 1991 impulsa el mejoramiento sistemático de las empresas a través del modelo de excelencia en gestión EFQM compuesto por nueve criterios: liderazgo, personas, política y estrategia, alianzas y recursos, procesos, resultados en las personas, resultados en los clientes, resultados en la sociedad y resultados claves. También existe la Fundación Premio Malcolm Baldrige, creada por un grupo de sobresalientes compañías de EE.UU., que diseñó en 1987 el Premio Malcolm Baldrige compuesto por siete criterios: liderazgo, planeación estratégica, enfoque de mercados y clientes, información y análisis, administración y desarrollo del recurso humano, administración de procesos y resultados de la empresa. De otra parte, la Unión de Científicos e Ingenieros Japoneses (JUSE, Union of Japanese Scientists and Engineers, por su sigla en inglés), estableció desde 1951 el Premio Deming integrado por 10 criterios: política y objetivos, organización y operación, educación y su diseminación, flujo de información y su utilización, calidad de producto y procesos, estandarización, gestión y control, garantía de calidad de funciones, sistemas y métodos, resultados y planes para el futuro. Por último, la Corporación Calidad creada en 1991 a través de esfuerzos mancomunados entre los sectores público y privado, diseña el Premio Colombiano a la Calidad de la Gestión, compuesto por ocho criterios: estrategia y gestión estratégica, liderazgo y estilo de gestión, gestión integral del talento humano, gestión de clientes y mercados, gestión de procesos, gestión del conocimiento y de la información, responsabilidad social y creación de valor y resultados. 
- Aspectos estadísticos de la calidad. Así como los aspectos administrativos logran exponer su orientación hacia el logro de resultados financieros de las empresas, los estadísticos también. Por ejemplo, el análisis de la calidad y su variación requieren de múltiples técnicas: los gráficos de control, de Pareto, histogramas, causa-efecto, entre otros. Todas ellas fundamentadas en el ejercicio de recolectar datos. Además, se usan otras técnicas como lluvia de ideas, los cinco por-qués, diagramas de afinidad, relaciones, árbol, 5w+2h, para complementar el análisis de calidad y formular su mejoramiento. Recientemente y basadas en las anteriores técnicas se han desarrollado diversas metodologías. Una muestra de ellas es el Seis Sigma, que se caracteriza por la rigurosidad en el uso de las técnicas y su énfasis en el logro de resultados concretos que ayuden al logro financiero.

El siguiente es un ejercicio que puede resumir cómo la dimensión técnica de la calidad influye en la creación de valor. Supongamos que una empresa dedicada a la producción de baterías presenta un valor medio de vida útil de 18 meses, una desviación estándar de dos meses y una garantía de 12 meses, y que el precio de venta de la batería es $\$ 200.000$ y la producción anual es de 100.000 unidades. Basados en el teorema del límite central, el porcentaje de reclamos de clientes es de $0.15 \%$, y el mismo porcentaje por pérdida por duración superior a 24 meses. Es decir, que la desviación estándar se debe reducir no sólo para disminuir el número de reclamos, sino también para aumentar la venta de productos porque la pérdida económica por falta de calidad equivale a \$60 MM anuales.

Este ejemplo demanda la realización de mejoras continuas en los diferentes tipos de procesos que realiza la empresa, porque el asunto es relevante tanto para la reducción de los costos por garantía como para aquellos de ventas por mayor duración a la esperada.

\subsection{Gestión del valor económico agregado (EVA)}

Entre los antecedentes del EVA se pueden señalar aquellos asociados con los citados por economistas neoclásicos Alfred Marshall entre lo más importantes que, refi- riéndose al concepto de generación de valor económico, hacen alusión a que el capital debería producir ingresos superiores respecto a su costo de oportunidad. En la década de 1920 la empresa General Motor Corporation adopta este concepto en el esquema de decisión de resultados; 30 años después lo retoma y comienza a utilizar un indicador asociado denominado ingreso residual. Ya en la década de 1980 y con la implementación de nuevos modelos económicos basados en la teoría del capital, las empresas se enfrentan a nuevos retos como el flujo mundial de capitales, la apertura de mercados y la desprotección del sector doméstico, las privatizaciones en aras de reducir el tamaño del Estado y la necesidad de mejores medidas de evaluación del desempeño empresarial, entre otros (Stewart, 2000).

EVA, entonces, está asociado con desafíos que propone la globalización y su competencia a nivel internacional, por cuanto ponen en riesgo el rendimiento económico de las empresas (Rivera, 2011) y la gran movilidad de capital dispuesto a ser invertido donde se ofrezcan mayores rentabilidades (García, 1998).

Ahora bien, EVA, indicador que hace referencia al valor económico de una empresa (con énfasis agregado), es una medida de la utilidad real después de deducir todos los costos que implica la operación de un negocio, incluido el costo de utilizar el capital aportado por los accionistas (Revista Semana, 1999); además su cálculo parte de las ventas, medida del efectivo que ingresa a la empresa por concepto de la venta de productos y servicios, y a estas se le restan los gastos incurridos en la operación, incluidos los impuestos que se deben pagar sobre las utilidades operacionales (NOPAT) Net Operating Profit After Taxes, sigla en inglés para el beneficio neto obtenido antes de los gastos financieros y después de impuestos, cifra a la cual hace falta determinar el costo de financiar el capital que usa la empresa. Entonces se toma un promedio ponderado del costo de la deuda y del capital y se multiplica por el capital total (deuda y patrimonio) que existe en el balance. EVA es el resultado de restarle este cargo de capital al NOPAT. Así, pues, EVA es una medida de la utilidad operacional después de impuestos y del costo del capital. 


\begin{tabular}{c|c}
\hline Información & 2013 proyección \\
\hline Ventas & $\$ 1.450 \mathrm{MM}$ \\
Costo de venta & $\$ 950 \mathrm{MM}$ \\
Gastos administrativos & $\$ 110 \mathrm{MM}$ \\
Gastos de ventas & $\$ 80 \mathrm{MM}$ \\
Impuestos & $\$ 60 \mathrm{MM}$ \\
Activo fijo neto & $\$ 350 \mathrm{MM}$ \\
Capital de trabajo neto & $\$ 650 \mathrm{MM}$ \\
Operativo KTNO & \\
Costo de & $25 \%$ \\
capital (CK) & \\
Rentabilidad del activo & $20 \%$ \\
neto & \\
\hline
\end{tabular}

Caso 1. EPC Ltda, es una empresa industrial que proyectó los siguientes indicadores financieros para el 2013:

- Se calcula EVA proyectado

$E V A=U O D I-(A C T I V O S$ NETOS DE OPERACIÓN) * CK)

\section{Paso 1. Calcular el UODI}

UODI = Utilidad Operativa - Impuestos

UO = Ventas - Costos - Gatos Operacionales

$U O=1450-950-110-80$

$U O=310 \mathrm{MM}$

$\mathrm{UODI}=310-60$

$\mathrm{UODI}=250 \mathrm{MM}$

\section{Paso 2. Calcular el activo neto de ope- ración}

$A N O=K T N O+A F N O+$ Otros activos operativos

$A N O=650+350+0$

$A N O=1.000 \mathrm{MM}$

\section{Paso 3. Calcular EVA}

$E V A=250.000 .000-(1.000 .000 .000 *$ 25\%)

$E V A=0 M M$
EVA es la utilidad que produce una empresa después de considerar todos los costos, incluidos los financieros y el costo de la inversión de los propietarios, en este caso la empresa no está proyectando para ese período determinado un EVA positivo. Esto puede estar asociado con diferentes factores, restricciones para lograr ahorros en costos de productos y en los gastos administrativos, debidos a la carencia de herramientas en gestión, de manera tal que sólo se está proyectando la utilidad mínima que debería obtener la empresa y no una rentabilidad superior al costo de capital.

Por otro lado, Stern, Stewar \& Co., firma propietaria de la marca EVA y que encierra dicho concepto, es quien sigue la idea de Marshall citado por Stewart (2000), sobre su teoría marginalista en donde señala "las ganancias contables no son suficientes si no son mayores que los costos de oportunidad del capital empleado" y la expande a través de procesos de consultoría empresarial a nivel mundial, orientando a las empresas hacia el objetivo básico financiero medido por EVA y dedicación de los recursos hacia la creación de valor económico.

Vale la pena señalar que los dos esquemas expuestos relacionados con la calidad efectiva operacional y EVA, involucran los conceptos de eficiencia operacional (EO) y eficiencia marginal (EM), los cuales tienen el propósito de analizar la eficiencia en los procesos o líneas de producción de una empresa, ante la variable de incremento o disminución de ventas. EO hace una comparación de la variación en costos y gastos respecto a la de los ingresos, exigiendo una mayor variación en los primeros respecto a las ventas. En el segundo caso (eficiencia marginal) se hace énfasis en los ingresos, variación que deberá ser superior a la de los costos y gastos de manera que la EM, sea positiva.

$E m=1-\frac{\Delta \text { Costos }+\Delta \text { Gastos }}{\Delta \text { Ingresos }} \quad E O=\frac{\Delta \text { Costos }+\Delta \text { Gastos }}{\Delta \text { Ingresos }}-1$

Para explicar mejor lo anterior, Rivera (2011) estableció un modelo de gestión basada en el valor (Gráfico 1), en el que a través de un conjunto de indicadores permite obtener el valor EVA. 


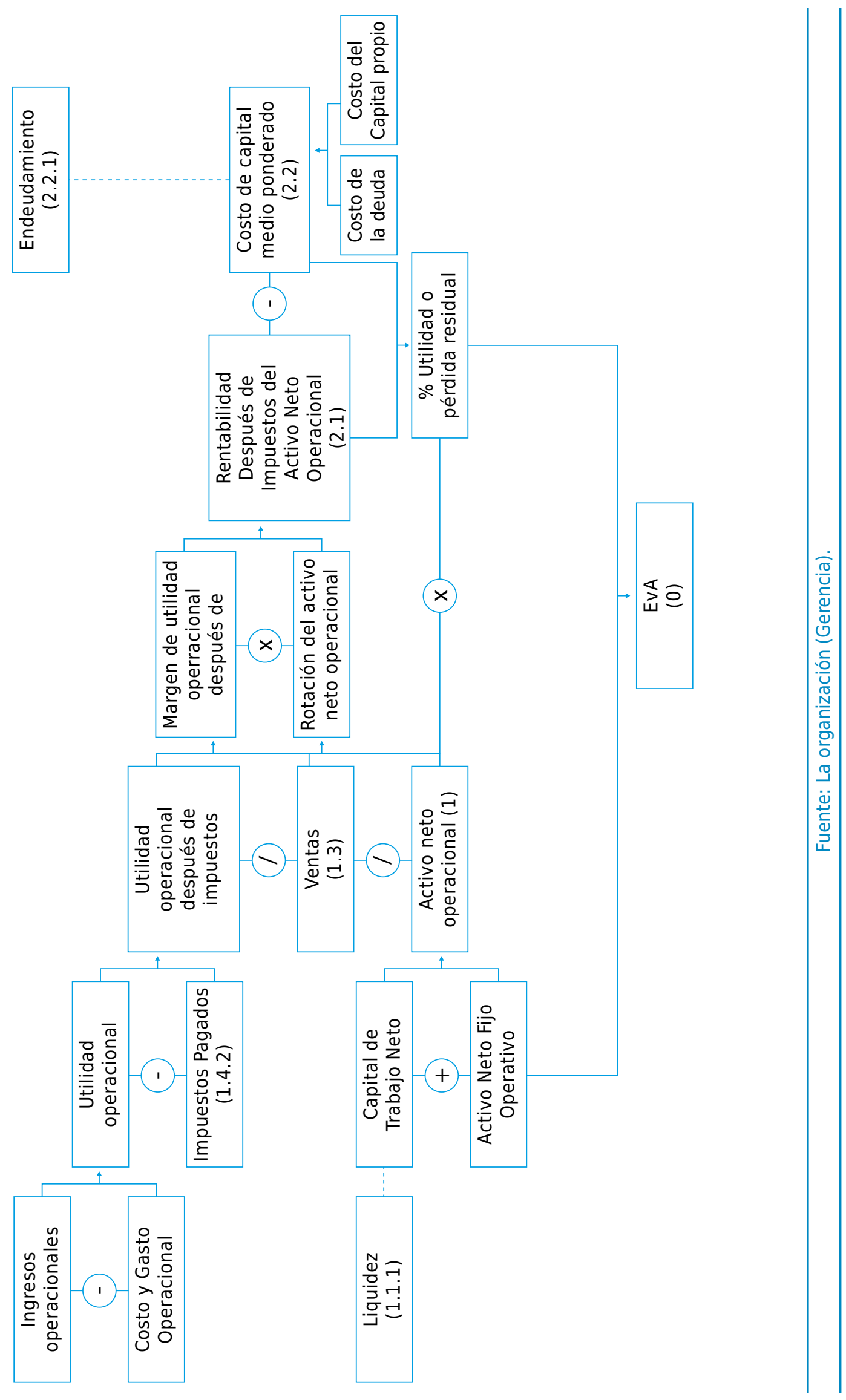


Lo anterior señala que en la medición del EVA se incluyen los resultados de esfuerzos internos y externos relacionados con los diferentes grupos de interés, porque, de acuerdo con la manera de calcular esta medida, a los ingresos se le restan todos los costos y gastos, que se deberán considerar con enfoque de inversión para no caer, como lo señala Wada (2001) en el concepto de pérdidas y ganancias (ventas menos costos: materiales, gastos, depreciación, salarios igual utilidad: antes de impuestos y dividendos). Entre los resultados que se incluyen para determinar la creación de EVA se debe tener en cuenta la eficiencia de procesos fundamentales como la operación, es decir, trabajar en la mejoría de la rentabilidad obtenida con el capital existente; optimizar el capital; la inversión, fijar la inversión en nuevos proyectos que generen una rentabilidad marginal superior; la liquidación, retirar la inversión que no se encuentra generando un retorno suficiente y está destruyendo valor en la empresa en conjunto; la financiación, reducir los costos de capital, estrategias que se revelarán en la creación de valor (García, 2003).

En la Tabla 1 se explican los indicadores asociados con EVA. Esto ayuda a entender mejor esta medida y facilita la identificación de los puntos en común (recuadros sombreados de los gráficos/modelos) con la calidad efectiva operacional (CEO), expuesta antes.

\section{Calidad efectiva operacional (CEO) ${ }^{2}$}

Para obtener una medida financiera de la calidad que integre la satisfacción del cliente y la reducción de la variación, aspectos que se han expuesto previamente, se ha construido el siguiente modelo que, mediante la combinación de indicadores del campo contable/financiera, sobre todo, permite obtener el valor de la CEO.

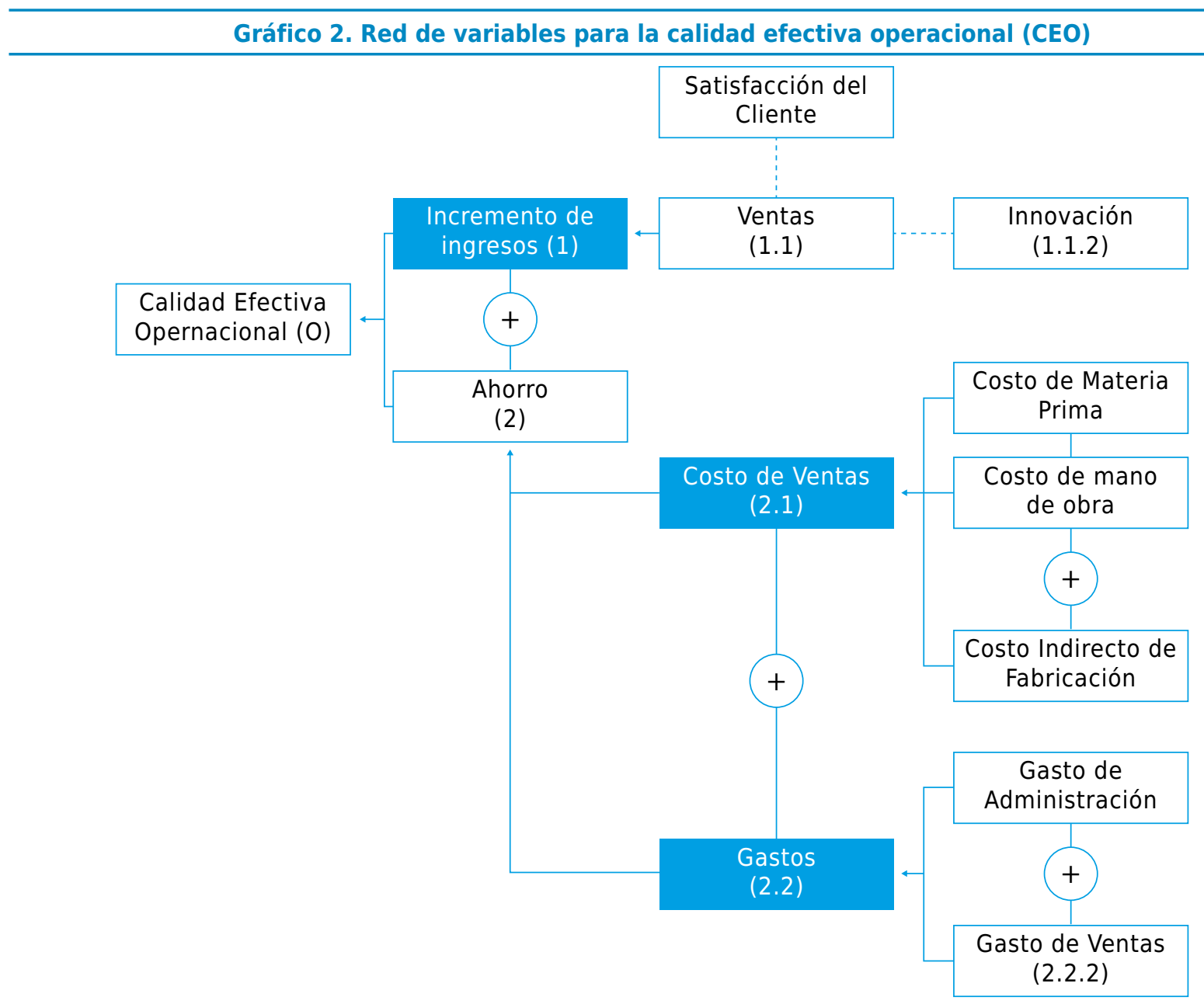

Fuente: Elaboración propia.

2 Término creado por el autor. 
Tabla 1. Indicadores para el economic value added

\begin{tabular}{|c|c|c|c|}
\hline $\mathbf{N}^{\circ}$ & Nombre & Concepto & Ecuación \\
\hline 0 & EVA & $\begin{array}{l}\text { Es la suma de la utilidad operativa después } \\
\text { de impuestos más el costo del capital medio } \\
\text { ponderado. }\end{array}$ & $\begin{array}{l}\text { EVA = Utilidad operativa después de impues- } \\
\text { tos - (activo neto operativo x costo del } \\
\text { capital medio ponderado). }\end{array}$ \\
\hline 1. & ANO & $\begin{array}{l}\text { Son los activos que están relacionados con } \\
\text { la actividad generadora de renta. }\end{array}$ & $\begin{array}{l}\text { ANO }=\text { Activos totales- activos no operativos } \\
\text { ANO }=\text { KTNO }+ \text { activos fijos }+ \text { otros activos } \\
\text { operativos no corrientes }\end{array}$ \\
\hline 1.1 & CTNO & $\begin{array}{l}\text { Cantidad de dinero o capital que la empresa } \\
\text { necesita para el desarrollo normal de su } \\
\text { actividad. }\end{array}$ & $\begin{array}{l}\text { CTNOO= Capital de trabajo neto operativo } \\
\text { (inventarios + cartera - cuentas por pagar). }\end{array}$ \\
\hline 1.1 .1 & Liquidez (L) & $\begin{array}{l}\text { Cualidad de convertir activos en dinero en } \\
\text { efectivo para solventar las necesidades de } \\
\text { corto plazo. }\end{array}$ & \\
\hline 1.2 & AFNO. & $\begin{array}{l}\text { Es el valor neto del activo fijo que se } \\
\text { encuentra operando. }\end{array}$ & $\begin{array}{l}\text { AFNO }=\text { Activo Fijo Total- Activos fijos no } \\
\text { operativos }\end{array}$ \\
\hline 1.3 & V. & $\begin{array}{l}\text { Son los recursos financieros percibidos por } \\
\text { la empresa, en el desarrollo de sus operacio- } \\
\text { nes principales. }\end{array}$ & $\begin{array}{l}\text { Ventas }(V)=(\text { Ingreso percibido por cada } \\
\text { unidad vendida). }\end{array}$ \\
\hline 1.4 & UODI & $\begin{array}{l}\text { Es el resultado de la operación, descontando } \\
\text { adicionalmente la proporción del estado. }\end{array}$ & $\begin{array}{l}\text { UODI= Utilidad operacional después de } \\
\text { impuestos (UO - impuestos) }\end{array}$ \\
\hline 1.4 .1 & UO & $\begin{array}{l}\text { Es el resultado económico de la operación } \\
\text { después de cumplir con las erogaciones de } \\
\text { producción, administración y comercialización. }\end{array}$ & $\begin{array}{l}\text { UO }=\text { Utilidad operacional (IO - CV - GA - } \\
\text { GV) }\end{array}$ \\
\hline 1.4.1.1 & 10 & $\begin{array}{l}\text { Ingresos percibidos de la actividad económica } \\
\text { principal de la empresa. }\end{array}$ & $\begin{array}{l}I O=\text { Ingreso operacional (valor de las ventas } \\
\text { de la actividad principal de la empresa) }\end{array}$ \\
\hline 1.4.1.2 & CGO & $\begin{array}{l}\text { Erogaciones y cargos destinados para el } \\
\text { desarrollo de la actividad económica principal. }\end{array}$ & $\begin{array}{l}\mathrm{CGO}=\text { Costo y gasto operacional }(\mathrm{CV}+\mathrm{GA} \\
+\mathrm{GV})\end{array}$ \\
\hline 1.4 .2 & IP & Valor proporcional de la utilidad. & $\begin{array}{l}\text { IP= Impuestos pagados (valor pagado por } \\
\text { impuestos). }\end{array}$ \\
\hline 2 & $\begin{array}{l}\text { Utilidad } \\
\text { residual-(UR) }\end{array}$ & & \\
\hline 2.1 & RDIANO & $\begin{array}{l}\text { Relación entre el rendimiento obtenido } \\
\text { después de impuestos con los activos } \\
\text { operacionales requeridos para operar. }\end{array}$ & $\begin{array}{l}\text { RDIANO = Rentabilidad después de impues- } \\
\text { tos del activo neto operacional }=(\text { UODI/A- } \\
\text { NO) }\end{array}$ \\
\hline 2.1 .1 & MUODI & $\begin{array}{l}\text { Porcentaje de utilidad operacional después } \\
\text { de impuestos. }\end{array}$ & $\begin{array}{l}\text { MUODI= Margen de utilidad operacional } \\
\text { después de impuestos (OUDI/V) }\end{array}$ \\
\hline 2.1 .2 & -RANO & $\begin{array}{l}\text { Mide la eficiencia de la empresa con la } \\
\text { administración y gestión de los activos } \\
\text { operacionales. }\end{array}$ & $\begin{array}{l}\text { RANO = Rotación del activo neto operacional } \\
\text { (V/Activo operacionales) }\end{array}$ \\
\hline 2.2 & $\begin{array}{l}\text { Costo de capital } \\
\text { medio } \\
\text { ponderado-CCMP }\end{array}$ & $\begin{array}{l}\text { Mide el promedio ponderado de los costos } \\
\text { de financiación. }\end{array}$ & $\begin{array}{l}\text { Tasa de interés de oportunidad o rentabili- } \\
\text { dad mínima requerida por el inversionista. }\end{array}$ \\
\hline 2.2 .1 & $\mathrm{E}$ & $\begin{array}{l}\text { Usar los recursos de terceros para financiar } \\
\text { una actividad y aumentar la capacidad de la } \\
\text { empresa. }\end{array}$ & $\begin{array}{l}\mathrm{E}=\text { Endeudamiento (pasivos totales/activos } \\
\text { totales) }\end{array}$ \\
\hline 2.2 .2 & $\begin{array}{l}\text { Costo de la } \\
\text { deuda(CD) }\end{array}$ & $\begin{array}{l}\text { Tasa de retorno del endeudamiento con } \\
\text { terceros. }\end{array}$ & \\
\hline 2.2 .3 & $\begin{array}{l}\text { Costo del capital } \\
\text { propio (CCP) }\end{array}$ & $\begin{array}{l}\text { Tasa de retorno que los inversionistas } \\
\text { requieren. }\end{array}$ & \\
\hline
\end{tabular}


El valor de CEO es la resultante de las medidas obtenidas de izquierda a derecha y aquellas que están sombreadas son las que más están ligadas con la medida del EVA como se observará en el modelo de gestión del valor.

En la Tabla 2 se describen los indicadores utilizados en la obtención de la medida de la calidad efectiva operacional-CEO-.

Caso 2. EPC Ltda.

Con la empresa EPC Ltda se realizó una proyección de ingresos y gastos para el año
2014, donde se contó sólo con la parte financiera y el histórico de la empresa. Con el propósito de cumplir la proyección y superarla se trabajó la herramienta de calidad efectiva operacional, cumpliendo la satisfacción del cliente y ahorrando operacionalmente en el producto. Ahora compararemos el real contra el proyectado con el propósito de calcular la CEO (Tabla 3).

- En el caso anterior se calcula el EVA Proyectado el cual dio: \$0 MM

Tabla 2. Indicadores para la calidad efectiva operacional (CEO)

\begin{tabular}{|c|c|c|c|}
\hline $\mathbf{N}^{\circ}$ & Nombre & Concepto & Ecuación \\
\hline 0 & $\begin{array}{l}\text { Calidad efectiva } \\
\text { operacional (CEO) }\end{array}$ & $\begin{array}{l}\text { Es la suma del ingreso y ahorro operacional } \\
\text { obtenido en la gestión de un sistema (empresa, } \\
\text { proceso). }\end{array}$ & $\begin{array}{l}\text { CEO = Incremento en los Ingresos operacio- } \\
\text { nales + Ahorro }\end{array}$ \\
\hline 1 & $\begin{array}{l}\text { Incremento en el } \\
\text { Ingreso } \\
\text { operacional(IO) }\end{array}$ & $\begin{array}{l}\text { Es la variación en el resultado económico de } \\
\text { la satisfacción del cliente. }\end{array}$ & $\mathrm{IIO}=$ Ingresos reales-Ingresos proyectados \\
\hline 2 & Ahorro (A) & $\begin{array}{l}\text { Es el resultado económico del uso a los } \\
\text { recursos tangibles e intangibles del sistema. }\end{array}$ & $\begin{array}{l}A=(\text { Estándar-Real). Equivale a la diferencia } \\
\text { entre el presupuesto y resultado }\end{array}$ \\
\hline 1.1 & Ventas (V) & $\begin{array}{l}\text { Son los recursos percibidos por la empresa } \\
\text { en el desarrollo de sus operaciones. }\end{array}$ & V $=$ (Ingreso percibido por cada unidad vendida) \\
\hline 1.1 .1 & $\begin{array}{l}\text { Satisfacción del } \\
\text { cliente (SC) }\end{array}$ & $\begin{array}{l}\text { Nivel de cumplimiento de las expectativas y } \\
\text { necesidades del cliente. }\end{array}$ & $\begin{array}{l}\mathrm{SC}=\text { Promedio ponderado del cumplimiento } \\
\text { de las expectativas y necesidades }\end{array}$ \\
\hline 1.1 .2 & Innovación (I) & $\begin{array}{l}\text { Proceso de obtención de nuevos productos o } \\
\text { procesos. }\end{array}$ & I= Número de productos o procesos nuevos \\
\hline 2.1 & $\begin{array}{l}\text { Costo de ventas } \\
\text { (CV) }\end{array}$ & $\begin{array}{l}\text { Inversión que se realiza con la expectativa } \\
\text { de obtener ingresos presentes y futuros. }\end{array}$ & $\begin{array}{l}\mathrm{CV}=\text { Erogaciones y cargos asociados a la } \\
\text { producción o adquisición de un bien o servicio }\end{array}$ \\
\hline 2.1 .1 & $\begin{array}{l}\text { Costo de materias } \\
\text { primas (CMP) }\end{array}$ & $\begin{array}{l}\text { Costo de materiales que se utilizan en el } \\
\text { proceso de producción, para usar en la } \\
\text { fabricación de bienes destinados a la venta. }\end{array}$ & $\begin{array}{l}\mathrm{CMP}=\text { Erogaciones y cargos asociados a la } \\
\text { compra de materiales }\end{array}$ \\
\hline 2.1 .2 & $\begin{array}{l}\text { Costo de mano de } \\
\text { obra (CMO) }\end{array}$ & $\begin{array}{l}\text { Costo de la fuerza laboral que está asociada } \\
\text { con la elaboración del producto. }\end{array}$ & $\begin{array}{l}\mathrm{CMO}=\text { Sueldos personal directamente } \\
\text { relacionados con el proceso productivo + } \\
\text { prestaciones sociales }+ \text { parafiscales }+ \\
\text { cargos adicionales }\end{array}$ \\
\hline 2.1 .3 & $\begin{array}{l}\text { Costos indirectos } \\
\text { de fabricación } \\
\text { (CIF) }\end{array}$ & $\begin{array}{l}\text { Costos de fábrica o planta, que no están } \\
\text { directamente asociados con el producto o } \\
\text { proceso. }\end{array}$ & $\begin{array}{l}\text { CIF = Materiales indirectos }+ \text { mano de obra } \\
\text { indirecta }+ \text { servicios y demás erogaciones } \\
\text { de la planta }\end{array}$ \\
\hline 2.2 & Gastos (G) & $\begin{array}{l}\text { Desembolsos de dinero no reembolsables, que } \\
\text { se llevan a cabo para la realización de las } \\
\text { operaciones de comercialización, administra- } \\
\text { ción, distribución, y financiación de la empre- } \\
\text { sa con el fin de realizar sus operaciones. }\end{array}$ & $\begin{array}{l}\text { G= GA + GV + Gastos no operacionales } \\
\text { (financiación o intereses) }\end{array}$ \\
\hline 2.2 .1 & $\begin{array}{l}\text { Gasto de } \\
\text { administración } \\
\text { (GA) }\end{array}$ & $\begin{array}{l}\text { Erogaciones indispensables para la función } \\
\text { administrativa. }\end{array}$ & $\begin{array}{l}\text { GA= Sueldos de personal de administración } \\
+ \text { papelería + impuestos + gastos legales + } \\
\text { servicios }\end{array}$ \\
\hline 2.2 .2 & $\begin{array}{l}\text { Gastos de ventas } \\
\text { (GV) }\end{array}$ & $\begin{array}{l}\text { Erogaciones ocasionales en la gestión de } \\
\text { ventas. }\end{array}$ & $\begin{array}{l}\mathrm{GV}=\text { Sueldos de personal de ventas }+ \text { arrenda- } \\
\text { mientos }+ \text { locales }+ \text { publicidad }+ \text { servicios } \\
\text { públicos en locales }+ \text { fletes de ventas }\end{array}$ \\
\hline
\end{tabular}




\begin{tabular}{c|c|c}
\hline \multicolumn{3}{c}{ Tabla 3. Información financiera de la empresa } \\
\hline Información & $\mathbf{2 0 1 4}$ real & $\begin{array}{c}\mathbf{2 0 1 4} \\
\text { proyección }\end{array}$ \\
\hline Ventas & $\$ 1.500 \mathrm{MM}$ & $\$ 1.450 \mathrm{MM}$ \\
Costo de venta & $\$ 900 \mathrm{MM}$ & $\$ 950 \mathrm{MM}$ \\
Gastos administrativos & $\$ 100 \mathrm{MM}$ & $\$ 110 \mathrm{MM}$ \\
Gastos ventas & $\$ 100 \mathrm{MM}$ & $\$ 80 \mathrm{MM}$ \\
Impuestos & $\$ 50 \mathrm{MM}$ & $\$ 60 \mathrm{MM}$ \\
$\begin{array}{c}\text { Activo fijo neto } \\
\text { Capital de trabajo veto } \\
\text { Operativo KTNO }\end{array}$ & $\$ 350 \mathrm{MM}$ & $\$ 350 \mathrm{MM}$ \\
$\begin{array}{c}\text { Costo de capital (CK) } \\
\begin{array}{c}\text { Rentabilidad del activo } \\
\text { neto }\end{array}\end{array}$ & $25 \%$ & $\$ 650 \mathrm{MM}$ \\
\hline
\end{tabular}

Fuente: Fuente EPC Ltda.

- Cálculo del EVA real 2014

$E V A=U O D I-(A C T I V O S$ NETOS DE OPERACIÓN) * CK

\section{Paso 1. Calcular el UODI}

UPDI = Utilidad Operativa - Impuestos

$U O=$ Ventas - Costos - Gastos - Gastos

Operacionales

$U O=1.500-900-200$

$U O=400 \mathrm{MM}$

$U O D I=400-50$

$U O D I=350 \mathrm{MM}$

Paso 2. Calcular el activo neto de operación

$A N O=K T N O+A F N O+$ Otros activos operativos

$A N O=650+350+0$

$A N O=1.000 \mathrm{MM}$

Paso 3. Calcular EVA

$E V A=350.000 .000-(1.000 .000 .000 * 25 \%)$

$E V A=100 \mathrm{MM}$

- Otra forma de calcular Eva es:

$E V A=A N O *(R A N-C K)$

$E V A=1.000 .000 .000 *(35 \%-25 \%)$

$E V A=100 \mathrm{MM}$

Con el proyectado no se obtuvo ningún EVA, lo que indica que el ahorro operacional de costos y gastos, después de aplicar en la empresa herramientas de gestión como la $\mathrm{CEO}$, brinda una mayor rentabilidad.

Como lo se aclaró en el caso anterior, el EVA es la utilidad que produce una empresa después de considerar todos los costos, inclui- dos los financieros y el costo de la inversión de los propietarios; con la información financiera real se obtuvo un EVA de \$100 MM, los cuales serían el valor económico agregado que está generando la empresa para ese período determinado; esta obtiene \$100 MM por encima de la utilidad mínima que debería alcanzar; lo cual está asociado con diferentes causas: ahorro en costos de los productos, ahorro en los gastos administrativos, se está siendo más eficiente operacionalmente, porque está cubriendo con todas las obligaciones y adicionalmente a eso, se está obteniendo una rentabilidad superior al costo de capital.

- Calcular CEO real de 2014

\section{Paso 1. Calcular el incremento de los ingresos}

$110=$ Ingresos reales - Ingresos proyectados

$$
\begin{aligned}
& 110=1.500-1.450 \\
& 110=50 \mathrm{MM}
\end{aligned}
$$

\section{Paso 2. Calcular el ahorro}

$A=(C V$ proyectado $-C V$ real $)+(G$ proyec tado - $G$ real)

$$
\begin{aligned}
& \mathrm{A}=(950-900)+(190-200) \\
& A=40 M M
\end{aligned}
$$

\section{Paso 3. Calcular el CEO}

$$
\begin{aligned}
& C E O=110+A \\
& C E O=50+40 \\
& C E O=90 M M
\end{aligned}
$$

El indicador de la CEO es resultado de la sumatoria de las variaciones de los ingresos operacionales (ventas) y el ahorro de los costos y gastos, dos vectores hacia donde los significados de la calidad se han dirigido. La empresa obtuvo un CEO de 90 MM (utilidad operacional adicional), por tanto se puede afirmar que la empresa realiza acciones para la satisfacción del cliente y el accionista, porque la variación en los ingresos fue positiva, porque se incrementó en $50 \mathrm{MM}$ y el ahorro en los costos y gastos también fue positiva con un valor de $40 \mathrm{MM}$. Es decir, se demuestra que la calidad es un medio efectivo para 
el logro de resultados económicos, interés común del accionista con respecto al EVA, indicador que exige la implementación de acciones que beneficien al cliente y hagan buen uso de los recursos de la empresa.

\section{Conclusiones}

En primera medida, la calidad fundamentada en la orientación que tienen los diferentes significados sí está dirigida hacia la gestión económica del valor agregado. Al aumentar el índice de satisfacción del cliente sobre el producto/servicio se produce un incremento en la probabilidad de consumo generando un aumento de los ingresos operacionales, y en segunda medida, al mejorar la calidad se obtendrá una mejor tasa de conformidad del producto/servicio, por tanto una mejor variación. Entonces, aumento de ingresos y menor desperdicio, causan una mejor CEO, haciendo que los costos y gastos operacionales y el costo de ventas se reduzcan y así aumente la utilidad operacional, variable con alta incidencia en NOPAT.

El indicador CEO se enfoca en la eficiencia del proceso operacional de la empresa, es decir, de la producción, administración y comercialización del producto, este refleja la proporción de utilidad superior que se está obteniendo al aplicar modelos de gestión basados en la calidad administrativa y técnica dentro de la parte operacional de la empresa. EVA evalúa adicional a lo anterior, las parte impositiva, financiera y el costo del capital.

A través del modelo CEO se hace explicita la demanda sobre la fuerza de los clientes en la gestión empresarial, porque si ella existe en un alto grado la calidad aumentaría sistemáticamente y el costo del capital propio de las empresas sería un valor razonable para todos los grupos de interés y con ello se obtendría mayor eficiencia interna. De otra parte, se requiere que las direcciones organizacionales rivalicen sin el interés de ser las dueñas mayoritarias del mercado (construcción de monopolios), pues, con ello se genera distorsión en el mismo y podría motivar la concentración de la riqueza y el debilitamiento en el mejoramiento continuo de la eficiencia interna.

Además, en la generación de EVA, uno de los vectores claves de este es el nivel de utilidad o pérdida residual que está sujeto al resultado de la comparación entre la rentabilidad después de impuestos del activo neto operativo y el costo de capital medio ponderado, este último influido por el costo de la deuda y el costo del capital propio, que es definido por el socio mediante mecanismos de comparación en el mercado sobre rendimiento del capital. Si el socio compara el rendimiento del capital en el mercado sin ningún tipo de consideración puede caer en el error de establecer tasas de costo de capital muy altas y con ello asfixiar la sostenibilidad del EVA. Es decir, la tasa del socio respecto al capital que este aporta, se debe comparar teniendo en cuenta similitudes de enfoque empresarial en donde para una relación armónica entre CFO y FVA es necesario orientar la empresa hacia el bienestar colectivo de todos los grupos de interés.

De acuerdo con el modelo para la gestión del valor propuesto por Rivera (2011) a través del cual se puede medir EVA y los planteamientos de García (1998), se puede reconocer que esta medida depende de variables que están bajo y fuera del control de la administración, por ejemplo la calidad. Por tanto, el modelo para medir la CEO resultaría de gran utilidad para las empresas que gestionan EVA.

Por su parte el valor de CEO depende de variables que están bajo el control único de la administración, que también son incluidas en el EVA. Es decir, que hacer esfuerzos para tener una mejor medida de CEO ayuda también a mejorar el valor del EVA y en sentido contrario. Con esto se logra demostrar que administrar una organización con enfoque en calidad sí debe arrojar resultados financieros satisfactorios para todos los grupos de interés.

\section{Referencias}

Aldana, L. Á. (2010). Administración por Calidad (1ª edi. cap. 1-3). Bogotá, Colombia: Editorial Alfaomega.

Centro Nacional de Productividad. (2005). Impactos de los sistemas de gestión de la calidad en las empresas colombianas. Cali, Colombia: Icontec.

Corporación Calidad. (2010). Empresas exitosas y sostenibles-Prácticas de clase mundial. Bogotá, Colombia: Corporación Calidad. 
Escalante, E. (2006). Análisis y mejoramiento de la calidad. (1ª̂edi. Cap. 2). México D.F., México: Editorial Limusa.

Deming, W. (1989). Calidad, productividad y competitividade: la salida de la crisis. Madrid, España: Editorial Diaz de Santos.

Feigenbaum, A. (1995). Control Total de la Calidad (3 edi.). México D.F., México: C.E.C.S.A.

García, O. L. (2003). Valoración de empresas, Gerencia de Valor y EVA (Cap. 1,5) .Medellín, Colombia. Editorial García, Oscar León.

García, A. (1998). Qué es y cómo medir el "EVA". Revista Dinero, (62), 20-26.

Guasch, L., Racine, J., Sánchez, I., y Diop, M. (2008). Sistemas de calidad y estándares hacia la construcción de ventaja competitiva (1 ${ }^{\mathrm{a}}$ ed. Cap. 1,2). Bogotá. Editorial Mayol Ediciones S.A.
Ishikawa, K. (1997). ¿Qué es el control total de la calidad? (Cap. 2-4). Bogotá, Colombia. Editorial Norma.

Rivera, J. (2011). Gestión basada en el valor. Cali, Colombia. Universidad del Valle.

Revista Semana. (1999, febrero). En busca de la utilidad real. Revista Semana, 69-71.

Stewart, G. (2000). En búsqueda del Valor. Barcelona, España: Editorial Gestión 2000.

Wada, K. (2001). Manual para la medición de productividad con enfoque de valor agregado. Cali, Colombia: CNP.

Walton, M. (1997). Cómo administrar con el método Deming (Cap. 4-20). Bogotá, Colombia: Editorial Norma. 